\title{
On the Semiclassical Impulse Approximation for Electron Capture in Asymmetric Ion-Atom Collisions
}

\author{
D.H. Jakubaßa-Amundsen ${ }^{\star}$ and P.A. Amundsen \\ Institute of Physics, University of Oslo, Norway
}

Received June 16, 1980

\begin{abstract}
A derivation of the impulse approximation for the capture of a target $K$-shell electron by a light projectile in ion-atom collisions is given in the framework of the semiclassical approximation. The impact-parameter dependence of the capture probability is calculated numerically without further approximations, and shows good agreement with recent experimental results for protons colliding with $\mathrm{Ne}$ and Ar. The validity of several peaking approximations and the relation to ionisation theories is briefly discussed.
\end{abstract}

\section{Introduction}

Charge transfer in fast collisions of heavy ions has long been a field of general interest both experimentally and theoretically. There has been a large number of experiments on total transfer cross sections, most of them concerning proton-hydrogen collisions (see i.e. $[1,2]$ and references quoted therein) and later also heavier systems [3-5]. In order to get more insight into the transfer process, also differential cross sections have been measured [6]. Recently, Horsdal Pedersen et al. [7] have made a systematic study of the impact-parameter dependence on various systems to obtain information about the influence of the system asymmetry and collision velocity on the capture process.

This calls on an effort to attempt a satisfactory theoretical description of both total cross sections as well as impact-parameter $(b)$ dependent capture probabilities. As it is well known that the first-order Brinkman-Kramers theory does not generally reproduce the absolute value of the experimental data [8] several higher-order theories have been suggested. Among them is the second-order Born approximation which has been shown to provide the dominant term at high collision velocities $v$ [9] but its evaluation is rather involved at moderate $v[10]$. Another approach that has been suggested is the continuum

* Supported by NORDITA

Present address: Physik-Department T30, Technische Universität München, D-8046 Garching, Reaktorgelände, Federal Republic of Germany distorted wave (CDW) approximation $[11,12]$ which pays particular attention to the correct asymptotic behaviour of the scattering states. It involves approximations, however, that lead to a dip in the $b$-distribution which is not experimentally observed. A related approach is the continuum intermediate state (CIS) approximation [13], but so far no impact-parameter distributions have been reported for this theory. Actually, the CIS approximation is related to the impulse approximation as shown in Appendix A. Also the semiclassical eikonal approximation has been used [14] which goes beyond second order as it includes the interaction between the transferred electron and the target to all orders. Although this theory is easy to handle even for the transfer to highly excited states, it provides only total cross sections and has not yet been extended to differential cross sections.

A well established method is the impulse approximation $[8,15]$. It may be derived by a systematic expansion of the Greens functions in terms of either of the two atomic potentials [16], thus being superior to the eikonal theory. By expanding in terms of the weaker potential this approximation should be valid in general for treating charge transfer from inner shells, not only for collision velocities much larger than typical orbital velocities of the transferred electron, but also for relatively slow collision systems, as long as they are sufficiently asymmetric. So far, calculations of the impulse approximation without any further simplifi- 
cation have only been carried out for proton-hydrogen and proton-helium collisions $[17,18]$, where the full quantum mechanical description is used. If one is, however, interested in the impact-parameter dependence, it is more convenient to start directly from the semiclassical impulse approximation (SCIA) which is equivalent to the quantum mechanical theory [16] as long as the classical description of the internuclear motion can be justified. In addition, one avoids the much discussed problem of considering explicitly the internuclear potential in the electronic transition amplitude [19]. As the SCIA is a theory which not only avoids spurious target-projectile overlap terms but also treats the nuclear and electronic motion completely separately, no internuclear potential occurs in the transition matrix elements whatsoever.

Calculations of the impact-parameter dependence of the capture probability from targets with charge $Z_{2}>2$ within the impulse approximation have until now only been carried out using an additional peaking approximation [20]. In the present paper we report calculations of the transfer probability from the $K$-shell of heavy atoms to protons, using the SCIA without any further approximations. As the derivation of the SCIA by Briggs [16] may be critisized for a somewhat careless treatment of the asymptotic behaviour of the electronic wavefunctions, and since the whole idea of using the impulse approximation for relatively slow, asymmetric collisions has recently been attacked [12], we give in Sect. 2 an alternative derivation of the SCIA. In Sect. 3 the transition amplitude for $K-K$ capture is evaluated, and several peaking approximations suggested in the literature are discussed (Sect. 4). Then follows in Sect. 5 a description of the capture into excited states. The scaling properties of the impulse approximation are investigated in Sect. 6. Numerical results for neon and argon targets are presented and compared with experiment and with the second Born approximation [21] as well as with the peaking approximations (Sect. 7). Since the theory for charge transfer is closely related to ionisation [22] as was pointed out by Briggs [16], and since both processes contribute to the creation of target $K$-shell vacancies, a comparison of the properties of both theories is given in Sect. 8. Some concluding remarks follow (Sect. 9). Atomic units $(h=m=e=1)$ are used throughout this paper unless otherwise indicated.

\section{The Semiclassical Impulse Approximation (SCIA)}

We formulate a derivation of the SCIA in order to stress the analogy between the impulse approxima- tion for charge transfer and the (first) Born approximation for ionisation, as both are the lowest-order terms in a systematic expansion of the transition amplitude after the weaker of the atomic potentials. The main difference between the two approaches is the asymptotic behaviour of the wavefunctions. For definiteness we shall consider transfer from a heavy target atom of charge $Z_{2}$ to a light projectile of charge $Z_{1}$.

In a coordinate system centered at the target atom the electronic Hamiltonian is in the one-electron approximation given by

$H=T+V_{2}(\mathbf{r})+V_{1}(\mathbf{r}-\mathbf{R})+V_{R}$

where $T$ is the kinetic energy, $V_{2}$ the target potential, $V_{1}$ the projectile potential and $V_{R}=-M_{1} /\left(M_{1}\right.$ $\left.+M_{2}\right) \mathbf{r} \ddot{\mathbf{R}}$ is the recoil potential which is present since the target atom is not an inertial frame of reference unless the classical path of the projectile, $\mathbf{R}(t)$, is a (constant-velocity) straight-line path (see i.e. [23]). $M_{1}$ and $M_{2}$ are the projectile and target mass, respectively.

The transition amplitude in the prior form is then given by [24]

$a_{f i}=-i \int_{-\infty}^{\infty} d t\left\langle\Psi_{f}^{(-)}(t)\left|V_{1}+V_{R}\right| \psi_{i}^{T}(t)\right\rangle$

where $\left|\psi_{i}^{T}(\mathrm{t})\right\rangle$ is an eigenstate of $H_{2}=T+V_{2}$, and $\left|\Psi_{f}^{(-)}(t)\right\rangle$ is that solution of the full scattering problem which asymptotically develops into an eigenstate of $H_{1}(t)=T+V_{1}(t)=U(t) H_{1}^{\prime} U^{+}(t)$. Here, $U(t)$ is the transformation from the projectile to the target rest frame, so that $H_{1}^{\prime}$ is the (time-independent) projectile Hamiltonian in its own rest frame. Asymptotically (and for a straight-line path, always) $U(t)$ is a Galilean transformation, and we can drop $V_{R}$ in the definition of the asymptotic states, as it vanishes identically for $t= \pm \infty$.

In the following we have found it convenient to work with time-dependent Greens functions (Feynman propagators) defined as a solution of

$\left(i \hat{c}_{\mathrm{t}}-H_{i}\right) G_{i}^{(-)}\left(t, t^{\prime}\right)=\delta\left(t-t^{\prime}\right) \mathbb{1}, \quad i=1,2$

with the appropriate boundary conditions, where $\mathbb{1}$ is the unit operator in Hilbert space. Under a timedependent unitary transformation $U(t), H_{1}^{\prime}$ transforms as

$H_{1}^{\prime} \rightarrow H_{1}(t)=U(t) H_{1}^{\prime} U^{+}(t)-i U(t) \hat{\partial}_{t} U^{+}(t)$

while the corresponding $G_{i}^{(-)}\left(t, t^{\prime}\right)$ transforms as

$G_{i}^{(-)}\left(t, t^{\prime}\right)=U(t) G_{i}^{(-) \prime}\left(t, t^{\prime}\right) U^{+}\left(t^{\prime}\right)$. 
Thus, if $\left|\psi_{f}^{P}(t)\right\rangle=U(t)\left|\psi_{f}^{P}(t)\right\rangle^{\prime}$ is the projectile bound state as seen in the target system, the state $\left|\Psi_{f}^{(--)}(t)\right\rangle$ is solution of

$\left|\Psi_{f}^{(-)}(t)\right\rangle=\left|\psi_{f}^{P}(t)\right\rangle+\int_{-\infty}^{\infty} d t^{\prime} G_{1}^{(-)}\left(t, t^{\prime}\right)$

$\left(V_{2}+V_{R}\left(t^{\prime}\right)\right)\left|\Psi_{f}^{(-)}\left(t^{\prime}\right)\right\rangle$.

Unfortunately, in the Coulomb case the existence of such an integral equation is not established. One should then take suitably screened potentials and only remove the screening after the calculation of the matrix elements. As in a real experiment the Coulomb forces will always be ultimately screened, such a procedure should indeed be justified.

We are now interested in a systematic expansion of (2.6) in terms of the weak potential $V_{1}$, but in such a way that the asymptotic behaviour of $\left|\Psi_{j}^{(-)}\right\rangle$, which obviously depends on $V_{1}$, is retained to each order. To do this, we note that we can expand $G_{1}^{(-)}\left(t, t^{\prime}\right)$ as

$G_{1}^{(-)}\left(t, t^{\prime}\right)=G_{0}^{(-)}\left(t, t^{\prime}\right)+\int_{-\infty}^{\infty} d t^{\prime \prime} G_{0}^{(\cdots)}\left(t, t^{\prime \prime}\right)$

$V_{1}\left(t^{\prime \prime}\right) G_{0}^{(-1}\left(t^{\prime \prime}, t^{\prime}\right)+\ldots$

where $G_{0}^{(-)}\left(t, t^{\prime}\right)$ is the free Greens function.

At this point it is convenient to restrict oneself to a constant-velocity straight-line path, $\mathbf{R}=\mathbf{b}+\mathbf{v} t$. This is allowed if the projectile only changes velocity and direction by a small amount during the transfer process. It should be kept in mind, however, that the Coulomb deflection effects are more complicated than in the case of ionisation [22], because in the present case the additionally appearing operator $U(t)$ in general will contain acceleration-dependent terms.

Inserting (2.7) into (2.6) we then find

$$
\begin{aligned}
& \left|\Psi_{f}^{(-)}(t)\right\rangle=\left|\psi_{f}^{P}(t)\right\rangle+\int_{-\infty}^{\infty} d t^{\prime} G_{0}^{(-)}\left(t, t^{\prime}\right) V_{2}\left|\Psi_{f}^{(-)}\left(t^{\prime}\right)\right\rangle \\
& +\int_{-\infty}^{\infty} d t^{\prime} \int_{-\infty}^{\infty} d t^{\prime \prime} G_{0}^{(-)}\left(t, t^{\prime \prime}\right) V_{1}\left(t^{\prime \prime}\right) \\
& G_{0}^{(-)}\left(t^{\prime \prime}, t^{\prime}\right) V_{2}\left|\Psi_{f}^{(-)}\left(t^{\prime}\right)\right\rangle+\ldots
\end{aligned}
$$

If we break off this expansion after the second term and replace $\left|\Psi_{f}^{(-)}\left(t^{\prime}\right)\right\rangle$ by $\left|\psi_{f}^{P}\left(t^{\prime}\right)\right\rangle$, we recover the second (impact-parameter) Born approximation. We then would discard terms which contain the weaker potential $\left(V_{1}\right)$ once, but the strong potential at least twice. This should thus be a good approximation for rapid collisions [21], but it becomes poor at lower velocities.

On the other hand, if we retain all powers of $V_{2}$ in (2.8), but still only the lowest-order term in $V_{1}$, we get an approximation which for sufficiently asymmetric systems $\left(Z_{1} \ll Z_{2}\right)$ should be reliable even for slow col- lisions (as long as purely molecular effects can be neglected). This indeed leads to the impulse approximation.

To show this, we define a lowest-order solution by

$|\psi(t)\rangle=\left|\psi_{f}^{P}(t)\right\rangle+\int_{\alpha_{i}}^{x_{i}} d t^{\prime} G_{0}^{(-)}\left(t, t^{\prime}\right) V_{2}\left|\psi\left(t^{\prime}\right)\right\rangle$.

Then $\left|\Psi_{f}^{(-)}(t)\right\rangle$ satisfies

$$
\begin{aligned}
& \left|\Psi_{f}^{(-)}(t)\right\rangle=|\psi(t)\rangle+\int_{-x}^{\infty} d t^{\prime} G_{2}^{(-)}\left(t, t^{\prime}\right) V_{1}\left(t^{\prime}\right) \\
& \left(\left|\Psi_{f}^{(-)}\left(t^{\prime}\right)\right\rangle-\left|\psi_{f}^{P}\left(t^{\prime}\right)\right\rangle\right) \\
& =|\psi(t)\rangle+\int_{-\infty}^{\infty} d t^{\prime} G_{2}^{(-)}\left(t, t^{\prime}\right) V_{1}\left(t^{\prime}\right) \\
& \left(|\psi(t)\rangle-\left|\psi_{f}^{P}\left(t^{\prime}\right)\right\rangle\right)+\ldots
\end{aligned}
$$

As the matrix element (2.2) already contains one power of $V_{1}$, the lowest-order contribution in $V_{1}$ is found if we replace $\left|\Psi_{f}^{(-)}(t)\right\rangle$ by $|\psi(t)\rangle$ in (2.2). So it only remains to solve (2.9), which is straightforward:

Since $\left|\psi_{f}^{P}(t)\right\rangle$ is a Galilean transformed projectile state, we have

$$
\begin{aligned}
& \left|\psi_{f}^{P}(t)\right\rangle=\int d \mathbf{k}|\mathbf{k}\rangle\left\langle\mathbf{k} \mid \psi_{f}^{P}(t)\right\rangle \\
& =\int d \mathbf{k}|\mathbf{k}\rangle e^{-i E_{f}(\mathbf{k}) t} e^{-i \mathbf{k b}} \varphi_{f}^{P}(\mathbf{k}-\mathbf{v}) \\
& E_{f}(\mathbf{k})=E_{f}^{P}+\mathbf{k} \mathbf{v}-v^{2} / 2, \quad \mathbf{v}=v \mathbf{e}_{z}
\end{aligned}
$$

where $\varphi_{f}^{P}(\mathbf{k}-\mathbf{v})$ is a projectile momentum-space wavefunction, and $E_{f}^{P}$ the energy of this state. The free Greens function $G_{0}^{(-)}\left(t, t^{\prime}\right)$ is simply

$G_{0}^{(-1}\left(t, t^{\prime}\right)=(2 \pi)^{-1} \int_{x}^{\infty} d \omega \frac{e^{-i \omega\left(t-t^{\prime}\right)}}{\omega-T-i \varepsilon}, \quad \varepsilon \rightarrow 0$

so that $(2.9)$ is readily solved by iteration as

$$
\begin{aligned}
& |\psi(t)\rangle=\int d \mathbf{k} \varphi_{f}^{P}(\mathbf{k}-\mathbf{v}) e^{-i E_{f}(\mathbf{k}) t} e^{-i \mathbf{k b}} \\
& {\left[|\mathbf{k}\rangle+\frac{1}{E_{f}(\mathbf{k})-T-i \varepsilon} V_{2}|\mathbf{k}\rangle+\ldots\right]}
\end{aligned}
$$

The quantity in the brackets is just the Neumann expansion of an off-shell target continuum wavefunction. The amount it is off-shell is

$\delta E=k^{2} / 2-E_{f}(\mathbf{k})=(\mathbf{k}-\mathbf{v})^{2} / 2-E_{f}^{P} \approx\left\langle V_{1}\right\rangle^{P}$

i.e., given by the strength of the weak potential. It is thus consistent to replace $E_{f}(\mathbf{k})$ in the brackets of (2.13) by $k^{2} / 2$. The difference $\delta E$ can eventually be absorbed in the next term of the expansion (2.10). In this manner one avoids the manipulations of the asymptotic states necessary in Briggs derivation (see the remarks following (2.24) of [16]). 
The quantity in the brackets in (2.13) can then be replaced by a target continuum wavefunction, and inserting this in (2.2) we find the standard result of the impulse approximation

$a_{f i}=-i \int_{-\infty}^{\infty} d t \int d \mathbf{k} e^{i\left(E_{f}(\mathbf{k})-E_{i}^{T}\right) t} e^{i \mathbf{k b}} \varphi_{f}^{* P}(\mathbf{k}-\mathbf{v})$

$\left\langle\psi_{\mathbf{k}}^{T}\left|V_{1}\right| \psi_{i}^{T}\right\rangle$

where $E_{i}^{T}$ is the energy of the initial target state. In this expression the matrix element $\left\langle\psi_{k}^{T}\left|V_{1}\right| \psi_{i}^{T}\right\rangle$ is the same as the one appearing in the first-order Born approximation for ionisation, weighted with the momentum distribution of the bound projectile state. This indicates that the range of validity of the impulse approximation (2.15) should indeed roughly coincide with that of the straight-line Born approximation for ionisation.

\section{Impulse Approximation for $K-K$ Capture}

In this section we specialise the transition amplitude (2.15) to the capture from a $1 \mathrm{~s}$ target state to the $K$ shell of the projectile. Making use of the Fourier representation of the projectile field

$V_{1}(\mathbf{r}-\mathbf{R})=-\frac{Z_{1}}{2 \pi^{2}} \int \frac{d \mathbf{s}}{s^{2}} e^{i \mathbf{s}(\mathbf{r}-\mathbf{R})}$

the time integral in (2.15) can be performed immediately, and we obtain

$a_{f i}=i Z_{1} / \pi \int d \mathbf{k} \varphi_{f}^{* P}(\mathbf{k}-\mathbf{v}) \int \frac{d \mathbf{s}}{s^{2}}$

$\cdot\left\langle\psi_{\mathbf{k}}^{T}\left|e^{i \mathbf{s r}}\right| \psi_{i}^{T}\right\rangle e^{i(\mathbf{k}-\mathbf{s}) \mathbf{b}}$

$\delta\left(\Delta E-v^{2} / 2+(\mathbf{k}-\mathbf{s}) \mathbf{v}\right)$

with $\Delta E=E_{f}^{P}-E_{i}^{T}$. For an initial hydrogenic $1 s$ state, the ionisation matrix element $\left\langle\psi_{k}^{T}\left|e^{i s r}\right| \psi_{i}^{T}\right\rangle$ can be evaluated analytically ([8], p. 364). In order to reduce the number of integrals in (3.2), we change variables from $\mathbf{k}$ to $\mathbf{q}_{0}=\mathbf{k}-\mathbf{s}$ and introduce spherical coordinates for $q_{0}$ and $\mathbf{s}$. Then the integrals over the angles $\vartheta_{q_{0}}$ (between $q_{0}$ and $\mathbf{v}$ ) and $\varphi_{q_{0}}$ can be performed immediately, leading to

$a_{f i}=2 i Z_{1} / v \int_{q_{\min }}^{\infty} q_{0} d q_{0} J_{0}\left(q_{0} b \sin \vartheta_{q_{0}}\right)$

$\int \frac{d \mathbf{s}}{s^{2}} \varphi_{f}^{* P}\left(\mathbf{q}_{0}+\mathbf{s}-\mathbf{v}\right)\left\langle\psi_{\mathbf{q}_{0}+\mathbf{s}}^{T}\left|e^{i \mathbf{s r}}\right| \psi_{i}^{T}\right\rangle$

where $q_{\min }=q_{0}\left|\cos \vartheta_{q_{0}}\right|=|-\Delta E / v+v / 2| . J_{0}$ is a Bessel function of the first kind. With the choice of these coordinates, the ionisation matrix element only depends on three variables
$\left\langle\psi_{\mathbf{q}_{0}+\mathbf{s}}^{T}\left|e^{i \mathbf{s r}}\right| \psi_{i}^{T}\right\rangle=c_{0} M\left(s, q_{0}, \cos \vartheta_{s}\right)$

$M\left(s, q_{0}, \cos \vartheta_{s}\right)=e^{\pi \eta / 2} \Gamma(1-i \eta) \frac{\left[s^{2}-\left(k+i Z_{2}\right)^{2}\right]^{-i \eta}}{\left(Z_{2}^{2}+q_{0}^{2}\right)^{2-i \eta}}$

$\left\{(1+i \eta) \frac{Z_{2}^{2}+q_{0}^{2}}{s^{2}-\left(k+i Z_{2}\right)^{2}}+1-i \eta\right\}$

$c_{0}=2^{3 / 2} Z_{2}^{5 / 2} / \pi, \quad \eta=Z_{2} / k$,

$k=\left|\mathbf{q}_{0}+\mathbf{s}\right|=\left(q_{0}^{2}+s^{2}+2 q_{0} s \cos \vartheta_{s}\right)^{\frac{1}{2}}$.

The integral over the angle $\varphi_{s}$ can also be performed analytically since $M$ is independent of $\varphi_{s}$. For a hydrogenic $1 s$ projectile state, one finds

$$
\begin{aligned}
& \int_{0}^{2 \pi} d \varphi_{s} \varphi_{f}^{* P}\left(\mathbf{q}_{0}+\mathbf{s}-\mathbf{v}\right) \\
& =2^{3 / 2} Z_{1}^{5 / 2} / \pi \int_{0}^{2 \pi} d \varphi_{s}\left(Z_{1}^{2}+\left(\mathbf{q}_{0}+\mathbf{s}-\mathbf{v}\right)^{2}\right)^{-2} \\
& =\left(2 Z_{1}\right)^{5 / 2} f_{0}\left(q_{0}, s, \cos \vartheta_{q_{0}}, \cos \vartheta_{s}\right) \\
& f_{0}=\alpha /\left(\alpha^{2}-\beta^{2}\right)^{3 / 2} \\
& \alpha=Z_{1}^{2}+q_{0}^{2}+v^{2}+s^{2}-2 q_{0} v \cos \vartheta_{q_{0}} \\
& +2 q_{0} s \cos \vartheta_{s}-2 v s \cos \vartheta_{q_{0}} \cos \vartheta_{s}
\end{aligned}
$$

and $\beta=-2 v s \sin \vartheta_{q_{0}} \sin \vartheta_{s}$. Writing $\cos \vartheta_{s}=x$, one arrives at the expression used in the numerical evaluation

$$
\begin{aligned}
& a_{f i}=32 i /(\pi v) Z_{1}^{7 / 2} Z_{2}^{5 / 2} \int_{q_{\min }}^{\infty} q_{0} d q_{0} J_{0}\left(q_{0} b \sin \vartheta_{q_{0}}\right) \\
& \int_{0}^{\infty} d s \int_{-1}^{1} d x M\left(s, q_{0}, x\right) f_{0}\left(q_{0}, s, \cos \vartheta_{q_{0}}, x\right) .
\end{aligned}
$$

Due to the complex structure of $M$ we did not find it possible to reduce the three integrals further. One should note that $M$ contains a square-root singularity at $k=0$ which arises from the normalisation factor of the Coulomb wave $\psi_{\mathbf{k}}^{T}$.

As the only impact-parameter dependence is contained in $J_{0}$, and the double integral over $M f_{0}$ is a smooth function decaying rapidly for large $q_{0}$, one expects $a_{f i}$ to vary smoothly with impact parameter. Only for very large velocities, corresponding to a large $q_{\min }$, wiggles appear due to the oscillatory behaviour of the Bessel function.

From (3.6) the total cross section is readily evaluated by making use of the orthogonality of the Bessel functions

$$
\begin{aligned}
& \sigma=2 \pi \int_{0}^{\infty} b d b\left|a_{f i}\right|^{2} \\
& =2 / \pi(32 / v)^{2} Z_{1}^{7} Z_{2}^{5} \int_{q_{\min }}^{\infty} q_{0} d q_{0} \\
& \left|\int_{0}^{\infty} d s \int_{-1}^{1} d x M\left(s, q_{0}, x\right) f_{0}\left(q_{0}, s, \cos q_{q_{0}}, x\right)\right|^{2} .
\end{aligned}
$$


This has to be multiplied by the number of initial electrons in the $K$-shell.

\section{Peaking Approximations}

In order to simplify the evaluation of the transition amplitude, several approximations have been introduced. In his original work, Briggs [16] suggests that in the starting expression (2.15) $\mathbf{k}$ may be replaced by $\mathbf{v}$ everywhere except in the Fourier transform $\varphi_{f}^{* P}(\mathbf{k}$ $-v)$ because the latter is strongly peaked at zero momentum. In this case, the $\mathbf{k}$ integral can be done trivially, yielding the wavefunction in the coordinate space at $\mathbf{r}=0$ :

$a_{f i}^{B}=-i(2 \pi)^{3 / 2} \psi_{f}^{* P}(0) \int_{-\tau}^{\infty} d t e^{i\left(\Delta E+v^{2} / 2\right) t}$

$\left\langle\psi_{\mathbf{v}}^{T}\left|V_{1}\right| \psi_{i}^{T}\right\rangle$.

This is indeed proportional to the ionisation amplitude leading to a final state with momentum $\mathbf{v}$ if the binding energy of the projectile state can be neglected in the energy phase. Actually, the formula (4.1) is limited to final $s$-states since $\psi_{f}^{* P}(0)$ vanishes otherwise. But in this case it is expected to be a reasonable approximation for very rapid collisions, because in this limit the internal momentum of the projectile state becomes negligible compared to $\mathbf{v}$.

A somewhat less restrictive peaking approximation has been considered by Bransden and Cheshire [17] who keep $\exp (i \mathbf{k} \mathbf{R})$ and the factor $\exp (-i \mathbf{k r})$ from the function $\psi_{\mathbf{k}}^{T}$ inside the $\mathbf{k}$ integral. Then

$$
\begin{aligned}
& a_{f i}^{B C}=-i e^{\pi \eta_{2} / 2}\left(1-i \eta_{2}\right) \int_{-a}^{\infty} d t e^{i\left(\Delta E+v^{2} / 2\right) t} \\
& \left\langle\psi_{f}^{P}(\mathbf{r}-\mathbf{R})_{1} F_{1}\left(-i \eta_{2}, 1,-i(v r+\mathbf{v} \mathbf{r})\right) e^{i \mathbf{v r}}\left|V_{1}\right| \psi_{i}^{T}\right\rangle
\end{aligned}
$$

with $\eta_{2}=Z_{2} / v$. This expression incorporates the additional $b$-dependence from (2.15). One may argue that the treatment of $\exp (-i \mathbf{k} \mathbf{r})$ on a different footing than the remainder of $\psi_{\mathbf{k}}^{T}$ is not justified except perhaps at very high velocities, because the confluent hypergeometric function ${ }_{1} F_{1}$ is also oscillating with $\mathbf{k}$. Actually, in the case of total cross sections the two peaking approximations (4.1) and (4.2) agree for asymmetric systems $Z_{1} / Z_{2} \ll 1[16]$.

Recently we have suggested a different peaking approximation which reduces the triple integral (3.6) to a double integral [20]. Starting from (3.2), the ionisation matrix element is taken outside the $\mathbf{k}$ integral at zero transverse momentum $\mathbf{k}_{\perp}$, whereas the $z$-component $k_{z}$ of the momentum transferred to the electron is correctly determined by the argument of the $\delta$-function in (3.2). This leads to

$$
\begin{aligned}
& a_{f i}^{I P}=16 i /(\pi v) b Z_{1}^{7 / 2} Z_{2}^{5 / 2} \\
& \int_{0}^{\infty} d s \int_{-1}^{1} d x J_{0}\left(s b \sqrt{1-x^{2}}\right) \frac{K_{1}\left(b \sqrt{Z_{1}^{2}+\left(q_{o z}-v\right)^{2}}\right)}{\left(Z_{1}^{2}+\left(q_{0 z}-v\right)^{2}\right)^{\frac{1}{2}}} \\
& e^{\pi \hat{\eta} / 2}(1-i \hat{\eta}) \frac{\left[s^{2}-\left(\left|q_{\mathrm{oz}}\right|+i Z_{2}\right)^{2}\right]^{-i \hat{\eta}}}{N^{2-i \hat{\eta}}} \\
& \left\{(1+i \hat{\eta}) \frac{N}{s^{2}-\left(\left|q_{\mathrm{oz} z}\right|+i Z_{2}\right)^{2}}+1-i \hat{\eta}\right)
\end{aligned}
$$

with $q_{\mathrm{oz}}=-\Delta E / v+v / 2+s x, \hat{\eta}=Z_{2} /\left|q_{\mathrm{oz}}\right|$ and $N=Z_{2}^{2}$ $+s^{2}+q_{\mathrm{oz}}^{2}-2 s \times q_{\mathrm{oz}}$. $K_{1}$ is a modified Bessel (Macdonald) function. Note that this expression also contains the square-root singularity (at $q_{\mathrm{oz}}=0$ ) as does the full theory. As discussed in [20], this peaking approximation is based on the fact that $\varphi_{f}^{* p}(\mathbf{q})$ is a rapidly decreasing function of $\mathbf{q}$, and that the ionisation matrix element is only weakly dependent on the transverse momentum. As will be discussed later, (4.3) is a good approximation for asymmetric systems and at high impact velocities.

\section{Capture into Excited States}

The approach of Sect. 3 is in principle easily extended to excited projectile states. The only change concerns the Fourier transform $\varphi_{f}^{* P}$ and thus the function $f_{0}$ appearing in the transition amplitude (3.6). Unfortunately, $f_{0}$ can apparently only be evaluated analytically for $m=0$ states. In case of the $2 s$ state, $f_{0}$ in (3.6) has to be replaced by

$f_{0} \rightarrow 2^{-3 / 2}\left(\hat{\alpha}^{2}-\beta^{2}\right)^{-5 / 2}\left[\hat{\alpha}\left(\hat{\alpha}^{2}-\beta^{2}\right)-Z_{1}^{2}\left(2 \hat{\alpha}^{2}+\beta^{2}\right) / 4\right]$

where $\hat{\alpha}$ and $\beta$ follow from the definitions below (3.5) with $Z_{1}$ replaced by $Z_{1} / 2$. For the $2 p, m=0$ state one finds

$f_{0} \rightarrow i Z_{1} 2^{-5 / 2}\left(\hat{x}^{2}-\beta^{2}\right)^{-5 / 2}$

$\left[\left(q_{0} \cos \vartheta_{q_{0}}-v+s \cos \vartheta_{q_{0}} \cos \vartheta_{s}\right)\left(2 \hat{\alpha}^{2}+\beta^{2}\right)\right.$

$\left.+3 /(2 v) \hat{\alpha} \beta^{2}\right]$.

If, however, the peaking approximation from [20] is introduced, an analytic evaluation is always possible. Indeed, as $Z_{1}<Z_{2}$, the asymmetry is effectively increased if one captures into higher states, such that the peaking approximation should be even more justified. In this approximation, it is the integral [20]

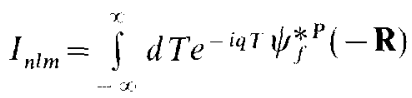

with $T=v t$ and $q=q_{\mathrm{oz}}-v$ that has to be evaluated (leading to the $K_{1}$ function of (4.3) in the $1 s$ case). It can be expressed by means of $K_{0}$ and $K_{1}$ functions for arbitrary final states. The general formula is given in Appendix B. 
The expressions (B.3)-(B.8) are very convenient for estimating the relative magnitude of the transition probability into the various projectile states. For velocities $v \sim v_{K}=Z_{2}$ the main contributions to the cross section come from $b \approx a_{K}=Z_{2}^{-1}, q \approx Z_{2}$ (thus $b q \approx 1$ and $Z_{1} \ll q$ ) such that for the $K$ and $L$ shell, $I_{n i m}$ can be approximated by

$I_{1 s} \approx \frac{2 b Z_{1}^{5 / 2}}{\pi^{\frac{1}{2}} q} K_{1}(b q), \quad I_{2 s} \approx \frac{b Z_{1}^{5 / 2}}{(2 \pi)^{\frac{1}{2}} q} K_{1}(b q)$

$I_{2 p, m=1} \approx \frac{b^{2} Z_{1}^{7 / 2}}{8 \pi^{\frac{1}{2}} q} K_{1}(b q)$,

$I_{2 p, m=0} \approx \frac{i b Z_{1}^{7 / 2}}{4(2 \pi)^{\frac{1}{2}} q^{2}}\left(2 K_{1}(b q)+b q K_{0}(b q)\right)$

and with $K_{0}(1) \approx K_{1}(1)$ the ratio between the total cross sections follows as

$\sigma_{1 s} / \sigma_{2 s} \approx\left(2^{3 / 2}\right)^{2}=8$

$\sigma_{2 p, m=0} / \sigma_{2 p, m=1} \approx(3 \sqrt{2})^{2}=18$

$\sigma_{2 p, m=0} / \sigma_{2 s} \approx\left(3 Z_{1} / 4 Z_{2}\right)^{2} \ll 1$.

The dominance of the capture into the ground state and the weak population of the states with higher angular momentum at velocities $v \gtrsim v_{K}$ has been pointed out repeatedly (see i.e. $[13,18]$ ) for symmetric systems. One should note, however, that this is no longer true if the asymmetry is reversed (i.e., $Z_{2} / Z_{1}$ $\ll 1)$. Then, due to resonance effects the population of higher $n$ and $l$ states may be enhanced $[14,25]$.

\section{Scaling Properties}

It has been shown that the Brinkman-Kramers theory obeys approximate scaling laws [26], and indeed the experimental data for charge transfer to protons are concentrated around a common curve for $v / v_{K} \gtrsim 1$ [3]. Therefore, it might be interesting to investigate the scaling properties of the impulse approximation.

To do this, we note that the ionisation matrix element (3.4) entering into the SCIA scales according to

$c_{0} M\left(s, q_{0}, \cos \vartheta_{s}\right)$

$=\left(2^{3 / 2} / \pi\right) Z_{2}^{-3 / 2} M\left(\hat{s}, \hat{q}_{0}, \cos \vartheta_{s} ; Z_{2}=1\right)$

if one introduces reduced momenta $\hat{s}=s / Z_{2}$ and $\hat{q}_{0}$ $=q_{0} / Z_{2}$. On the other hand, $\cos \vartheta_{q_{0}}$ contained in the lower integration boundary $q_{\min }$ can be expressed as

$\cos \vartheta_{q_{0}}=\frac{-\Theta_{12} / 2+\hat{v}^{2} / 2}{\hat{q}_{0} \hat{v}}=\frac{-1+\tilde{v}^{2}}{2 \tilde{q}_{0} \tilde{v}}$ where the momenta are now reduced by $Z_{2} \Theta_{12}^{\frac{1}{2}}$, i.e., $\tilde{v}=v /\left(Z_{2} \Theta_{12}^{\frac{1}{2}}\right)$ with $\Theta_{12}=2 \Delta E / Z_{2}^{2}$ which incorporates the deviation of the experimental binding energies from the hydrogenic ones. The difference between the reduced quantities in (6.1) and (6.2) does not allow for an exact scaling. For asymmetric systems, however, $\Theta_{12} \approx-2 E_{i}^{T} / Z_{2}^{2} \equiv \Theta_{2}$ depends only on the target. As $\Theta \frac{1}{2} \sim 0.8-0.9$ for the $K$-shell of all nonrelativistic targets with $Z_{2} \gtrsim 6$ is a slowly varying function of $Z_{2}$, the impulse approximation scales approximately as

$\sigma\left(v, Z_{1}, Z_{2}\right) \approx Z_{2}^{-2}\left(Z_{1} / Z_{2}\right)^{7} \hat{\sigma}\left(v / Z_{2}, Z_{1} / Z_{2}, Z_{2}=1\right)$

which is the better satisfied, the higher $v / v_{K}$, since then also the target binding energy can be neglected. The dependence of $\hat{\sigma}$ on $Z_{1} / Z_{2}$ enters only through the function $f_{0}$ in (3.5) and is roughly given by $\hat{\sigma} \sim\left(Z_{1} / Z_{2}\right)^{-2}$. This indicates an increase of the $K$ shell capture with approximately $Z_{1}^{5}$ and a decrease with $Z_{2}^{-7}$ at fixed reduced velocity $v / Z_{2}$ (like the Brinkman-Kramers result).

One may also derive approximate scaling laws for the impact-parameter dependent transition probability. As $b$ scales like an inverse momentum, one finds

$P\left(b, v, Z_{1}, Z_{2}\right) \approx\left(Z_{1} / Z_{2}\right)^{7} \hat{P}\left(b Z_{2}, v / Z_{2}, Z_{1} / Z_{2}, Z_{2}=1\right)$

where again the dependence of $\hat{P}$ on $Z_{1} / Z_{2}$ enters only through $f_{0}$.

\section{Numerical Results}

We have evaluated $1 s-1 s$ transfer probabilities and cross sections numerically from (3.6). The presence of a triple numerical integral and the (integrable) singularity at $k=0$ makes this somewhat tricky. In all integrations we have used a fifth-order recursive procedure, and by carrying out the $x$ integration of (3.6) first (which includes the singularity), the remaining integrands become fairly well-behaved, at least for collision velocities not too far from the target $K$ velocity. We feel confident that the numerical accuracy of the results presented here is about $5 \%$, and it can easily be improved by increasing the computer time. This accuracy is substantiated by comparison with calculations of the impact-parameter distribution in the quantal impulse approximation for $(\mathrm{p}, \mathrm{H})$ collisions reported by Coleman et al. [18], and by comparison between the total cross sections calculated directly from (3.7) and those obtained by numerical integration over impact parameters.

As the theory of Sect. 3 is restricted to hydrogenic electron wavefunctions, one has to face the question of how realistic such wavefunctions are. Indeed it is known from ionisation theories that the ionisation 
matrix element and thus also the ionisation probability even for $K$-shell ionisation is quite sensitive to the choice of wavefunctions, in particular for light atoms [27]. However, by using Slater screened hydrogenic wavefunctions and experimental binding energies, we estimate our results to be accurate within $20 \%$ for neon targets (possibly much better in some cases) and somewhat more accurate for argon.

We have not attempted to include contributions from excited final (projectile) states in our calculations, as they are small, and in view of the uncertainties in the choice of electronic wavefunctions, the large numerical effort needed to calculate them does not seem justified. Thus, according to the results of Sect. 5, our numerical values are probably systematically about $10 \%$ too low, when compared with the available experimental results.

For the comparison of our impact-parameter distributions with the experimental data we have used the simple semiclassical relation

$P(b(\vartheta))=\left(\frac{d \sigma}{d \Omega}\right)_{\mathrm{EC}} /\left(\frac{d \sigma}{d \Omega}\right)_{\mathrm{el}}$

where $(d \sigma / d \Omega)_{\mathrm{EC}}$ is the experimental differential capture cross section, $(d \sigma / d \Omega)_{\mathrm{el}}$ the atomic elastic scattering cross section and $b(\vartheta)$ the corresponding classical relationship between scattering angle and impact parameter. The theoretical transfer probability $P(b)$ from an initially full $K$-shell is simply given by

$P(b)=2\left|a_{f i}\right|^{2}$.

Equation (7.1) is applicable as long as the transfer amplitude does not vary rapidly with impact parameter (more precisely, with projectile angular momentum). Indeed, the differences between the results calculated from (7.1) and (7.2) and those calculated on the basis of an eikonal-type argument [28] are completely negligible for the systems discussed in the present paper.

In Fig. 1 we show the total cross sections for $K$-electron capture from neon. It is seen that for $v \gtrsim v_{K}$ the SCIA reproduces the experimental data quite well. The deviations at lower velocities may, at least partly, be due to the choice of simple wavefunctions. Also shown is the peaking approximation (4.3) which indeed agrees very well with the full calculation (3.6) at the higher velocities.

Figure 2 shows the scaled experimental cross sections for the $K$-electron capture from a target with $6 \leq Z_{2} \leq 18$ by a proton. As $\Theta_{2}$ is roughly constant in this region, it makes no difference to use the scaling from (6.2) instead of (6.3). Also shown is the scaled SCIA from Fig. 1 where we chose $\Theta_{2}^{\frac{1}{2}}=0.82$ corresponding to a neon target. There is good agreement

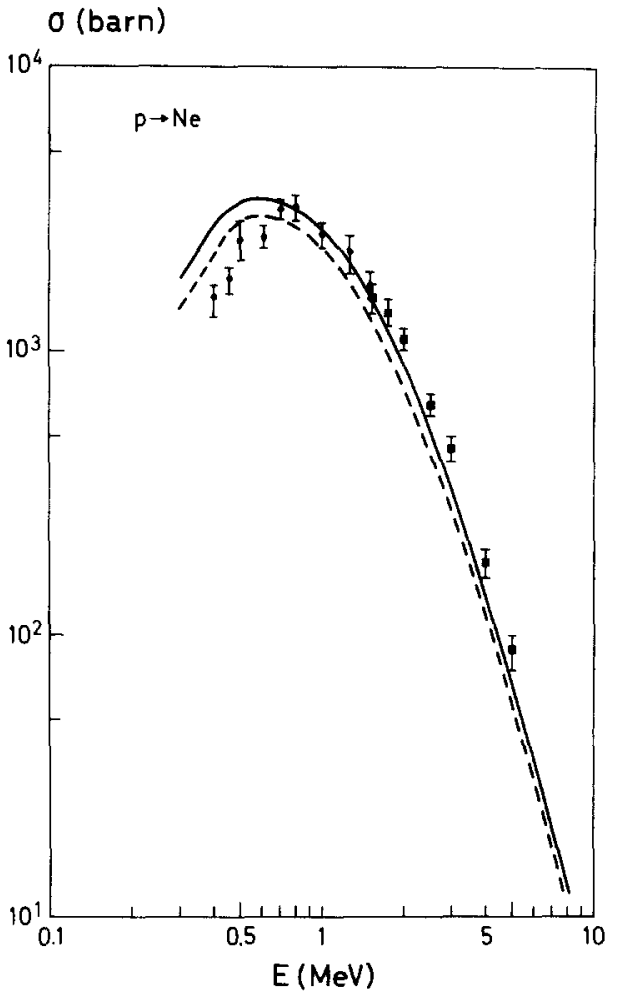

Fig. 1. Cross section for the capture of Ne $K$-shell electrons by protons as a function of projectile energy. The full curve denotes the SCIA (without approximation) and the broken curve shows the SClA-peaking (4.3). The data are from Rodbro et al. (\$) [3] and from Cocke et al. (1) [5]

with the experimental data for $v / v_{K} \gtrsim 1$, while the Brinkman-Kramers result (also shown) is systematically too large.

In Fig. 3 the impact-parameter dependence of the capture probability from $\mathrm{Ne}$ is given. The decrease with $b$ is monotonic and in agreement with the experimental fall-off. Also the peaking result (4.3) describes the $b$-dependence well, though the decrease for large $b$ is somehow too large for the lower velocities. At higher velocities, the peaking approximation gives the same slope. Also shown is Briggs' [16] peaking approximation (4.1). Whereas the slope of the impact-parameter distribution is described well for all cases, the absolute values are systematically too high except for very high velocities. One should note that the difference between the two peaking approximations is the appearance of $\Psi_{f}^{* P}(-\mathbf{R})$ in the former, while in (4.1) it is replaced by $\psi_{f}^{* P}(0)$, indicating that (4.1) is only valid if the interaction region is very narrow compared to the width of $\psi_{f}^{*^{P}}(-\mathbf{R})$. Thus the restrictions on $Z_{1} / Z_{2}$ and $v / v_{K}$ for Briggs' approximation are much stronger than for the applicability of (4.3).

Figure 4 shows the results for an argon target. The impact velocity of $1.7 \mathrm{MeV}$ is chosen such that it 


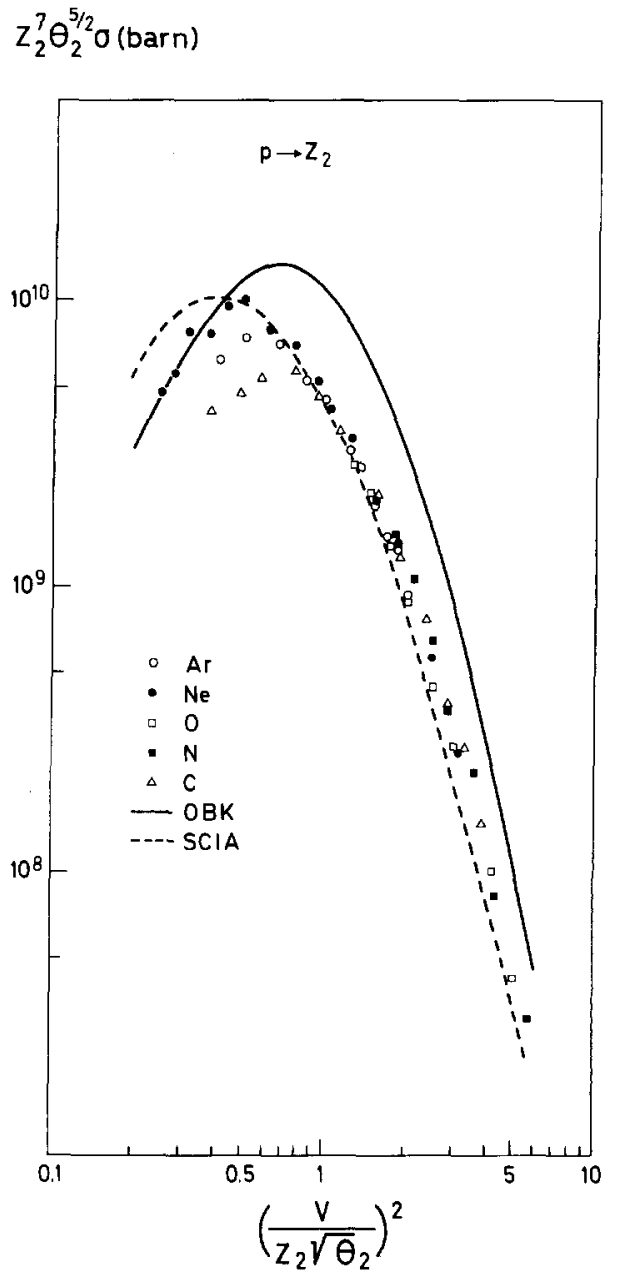

Fig. 2. Scaled cross section for the electron capture by protons from the $K$-shell of various target atoms as a function of the scaled velocity. The full curve is the Brinkman-Kramers result (taken from [3]), and the broken curve is the SCIA for Ne (without approximation). Experimental data are from [3] $(C, \mathrm{Ne}$ at $E \leq 1.5 \mathrm{MeV})$, from [5] $(\mathrm{O}, \mathrm{N}, \mathrm{Ne}$ at $E \geq 1.5 \mathrm{MeV})$ and from [4] (Ar)

yields the same ratio $v / v_{K}$ as for the $0.5 \mathrm{MeV}(\mathrm{p}, \mathrm{Ne})$ collision. For this more asymmetric system the agreement between experiment, the full SCIA and the peaking approximation (4.3) is even better. So one can conclude that for these asymmetric systems this peaking result is sufficiently good to explain the data within the experimental uncertainty. As (4.3) contains only two integrals, the integration technique has not to be so refined, and one saves computer time.

In Fig. 5 we have plotted the ratio between the SCIA and the corresponding Brinkman-Kramers result. This ratio falls with increasing velocity to about 0.2 and then increases again for very high velocities because the second-order term in the Born series (which is included in the SCIA, cf. Sect. 2) falls slower with velocity than the first-order term $[9,16]$. It should be noted that at low velocities $\left(v<v_{K}\right)$ the Brinkman-
$P(b)$

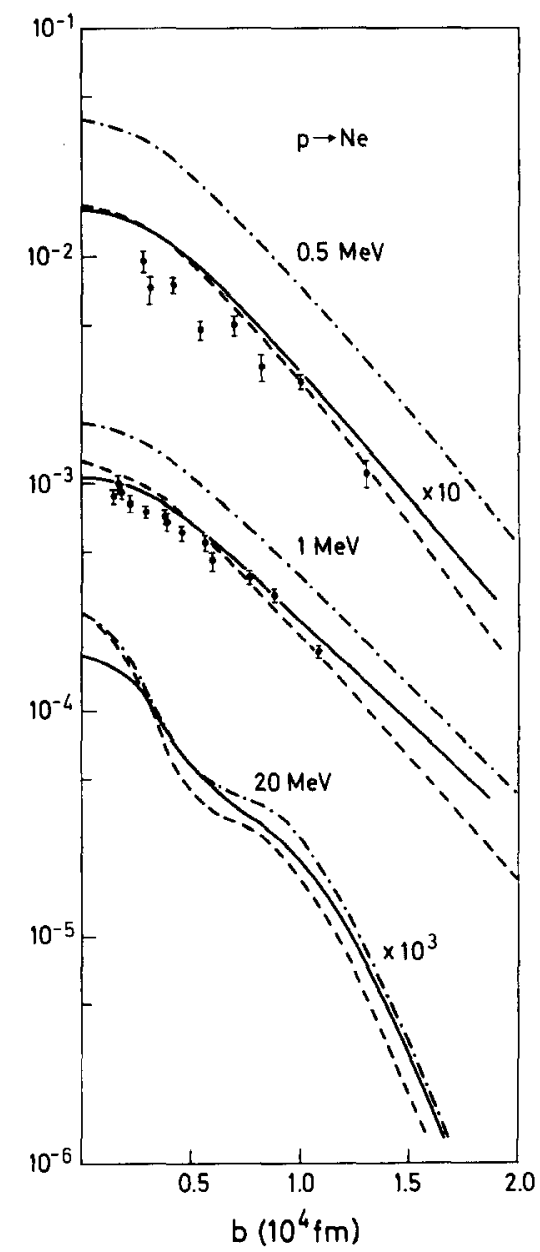

Fig. 3. Charge transfer probability for the capture of $\mathrm{Ne} K$-shell electrons by protons as a function of impact parameter at projectile energies $0.5,1$ and $20 \mathrm{MeV}$. The full curves are SCIA calculations (from (3.6)), the broken curves show the SCIA-peaking (4.3) and the chain curves denote the Briggs-peaking (4.1). Experimental data are from [7]

Kramers theory underestimates the capture probability. Also shown is the ratio calculated with the peaking approximation (4.3), which is easier to handle at high velocities than the full SCIA. The wiggles at the lower velocities indicate the break-down of the peaking approximation.

As the second-order Born approximation provides the correct asymptotic behaviour, it has also been used to calculate charge transfer processes $[10,21]$. In order to reduce the numerical work, Briggs and Dubé [21] introduced two additional peaking approximations, which further restrict the applicability, so that their results is only valid if $v$ is very much larger than $v_{K}$. In Fig. 5 we also show the ratio of this peaked second Born result to the Brinkman-Kramers formula. It is seen that for very high energies $\left(v / v_{K}\right.$ $\gg 1)$ the impulse approximation and the second 


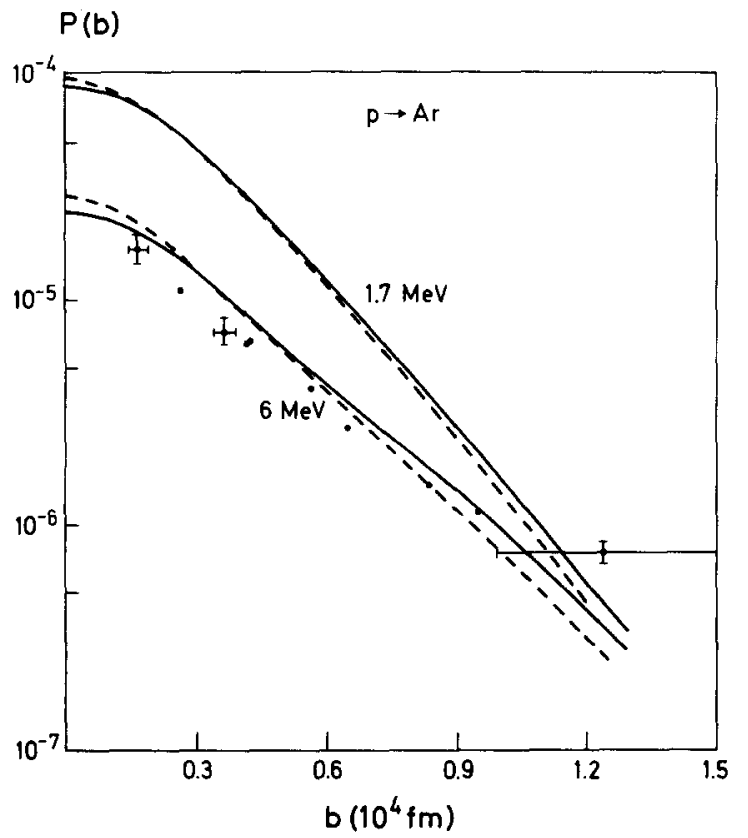

Fig. 4. Charge transfer probability for the capture of Ar $K$-shell electrons by protons as a function of impact parameter at projectile energies 1.7 and $6 \mathrm{MeV}$. The full curves are SCIA calculations (from (3.6)) and the broken curves show the SCIA-peaking (4.3). Experimental data are from [6]

Born theory do indeed seem to approach the same limiting value, as expected.

\section{Comparison with $K$-Shell Ionisation}

As pointed out before, there is a close connection between electron capture and ionisation in the structure of the transition amplitude. Impact parameter distributions can be characterised by their width which is a measure for the average internuclear distance at which the reaction takes place. In deeply inelastic reactions this width is simply given by the minimum momentum transfer during the collision, that is

$\Gamma_{\mathrm{ion}} \approx q_{\mathrm{ion}}^{-1} \approx \frac{v}{\Delta E} \approx \frac{v}{\left|E_{i}^{T}\right|}$

$\Gamma_{\mathrm{EC}} \approx q_{\mathrm{EC}}^{-1} \approx \frac{v}{\Delta E+v^{2} / 2}$

for ionisation and capture, respectively. It is in both cases determined by the energy difference of the electron between its final and initial state. On the other hand, the width of the $b$-distribution is also limited by the spatial extent of the initial state wavefunction, which is given by the $K$-shell radius $a_{K}$. This means that the relations (8.1) are only valid if $\Gamma \lesssim a_{K}$. Thus for ionisation, (8.1) is only meaningful for small velocities while for charge transfer it is also applicable for high velocities since $q_{\mathrm{EC}}^{-1}$ again decreases with $v$.

In Fig. 6 we compare the relations (8.1) with the width $\Gamma$ extracted from the $b$-distribution for both ionisation and electron capture. For the calculation of ionisation, the straight-line SCA is used [22] with the same wavefunctions and energies as for charge transfer. $\Gamma$ is calculated by means of $\Gamma=\bar{b}$ with $\bar{b}$ from $P(\bar{b})=\frac{1}{2} P(0)$. While the impact-parameter dependence is similar for both processes when $v / v_{K} \ll 1$, the ionisation probability decreases much slower with $b$ than the capture probability when $v$ becomes larger than $v_{K}$. Actually, for fast collisions charge transfer is more comparable to ionisation into a final state of a (large) definite energy $v^{2} / 2$ (cf. [16] and (4.1)).

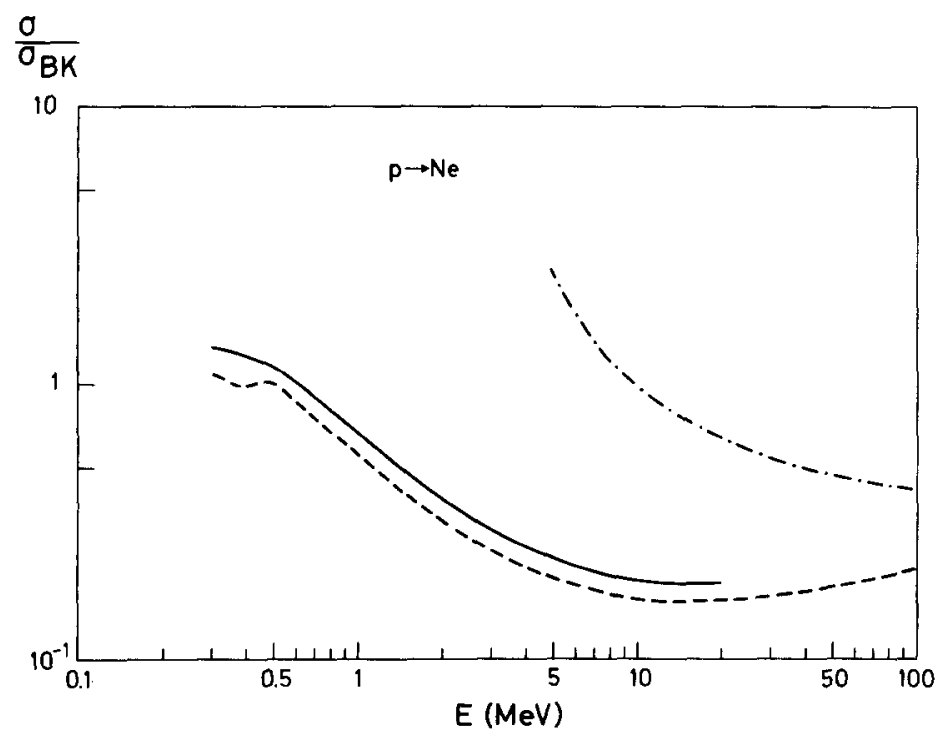

Fig. 5. Ratio of the cross sections for the capture of a Ne $K$-shell electron by protons evaluated with different theories to the Brinkman-Kramers result $\sigma_{B K}$ as a function of projectile energy. The full curve is calculated with the SCIA (3.6), the broken curve with the SCIA-peaking (4.3) and the chain curve with the peaked second-order Born approximation [21] 


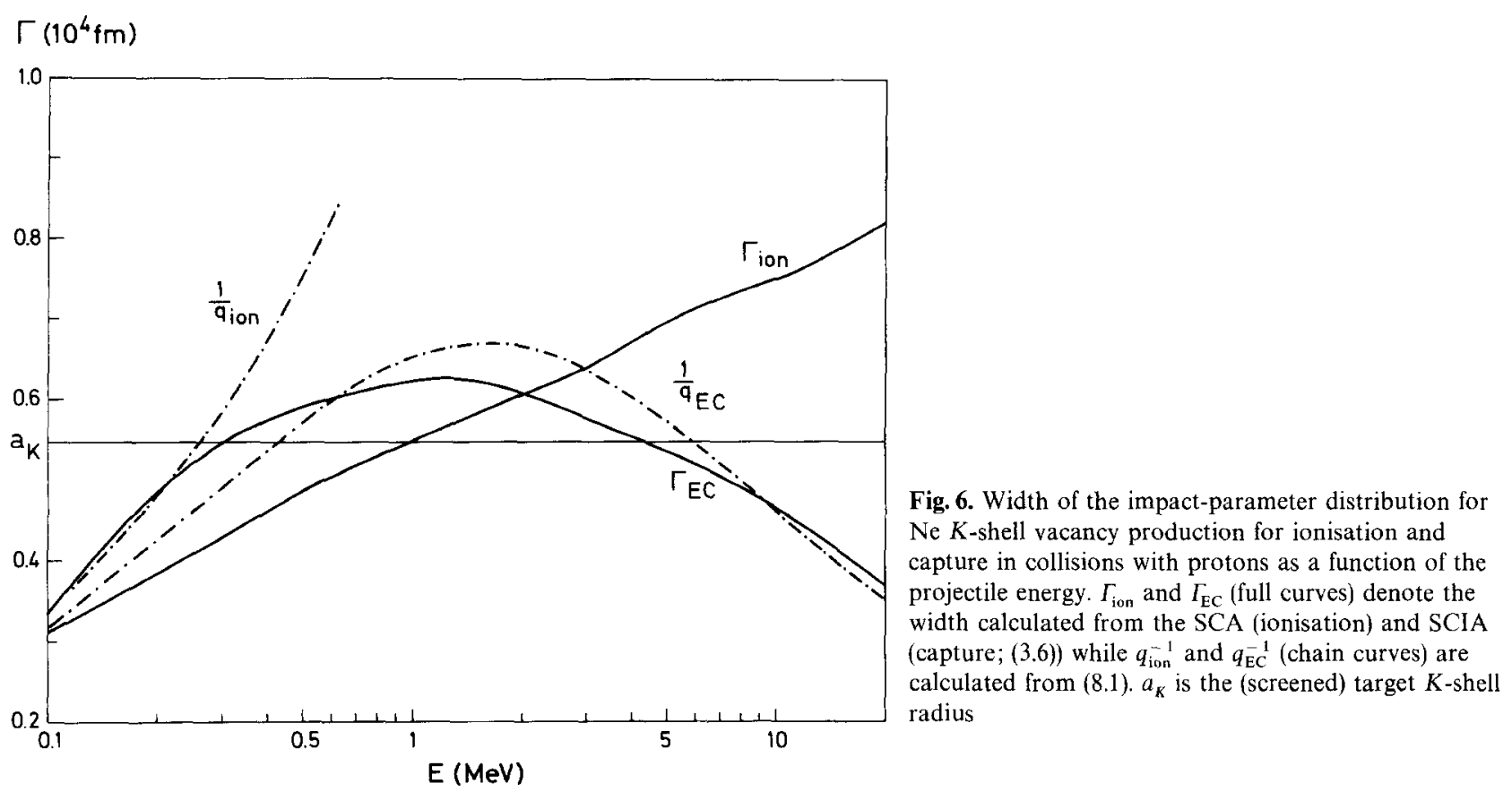

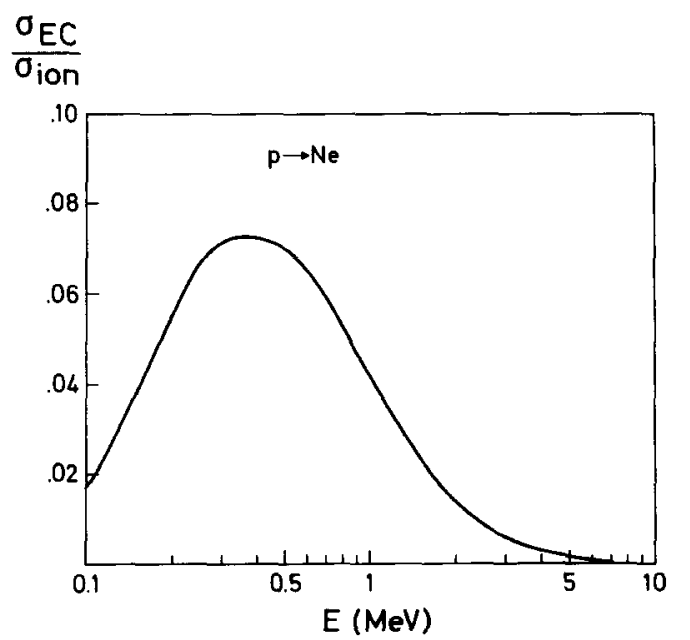

Fig. 7. Ratio of the cross sections for the capture of a $\mathrm{Ne} K$-shell electron $\left(\sigma_{\mathrm{EC}}\right)$ to the $K$-shell ionisation of $\mathrm{Ne}\left(\sigma_{\text {ion }}\right)$ by proton impact as a function of the projectile energy. $\sigma_{\mathrm{EC}}$ and $\sigma_{\text {ion }}$ are calculated with the SCIA (3.6) and SCA, respectively

As the probability of populating such a high-lying state is small compared to the total ionisation probability, the $K$-vacancy production from capture is much lower than from ionisation. This is illustrated in Fig. 7 where the cross section ratio between capture and ionisation is plotted for protons impinging on Ne. The maximum of this ratio is less than $10 \%$ for this system. For high velocities, the capture shows a rapid fall-off (cf. Fig. 1) and becomes negligible compared with ionisation. Also for low velocities the importance of capture is seen to become small. At these low velocities one must of course eventually take into account terms beyond the lowest order in the projectile field, as is the case for ionisation theories. To what extent, if any, such corrections can be incorporated in the simple SCIA-picture is not yet clear. All that can be safely said at present is that for a given $v / v_{K}$, the SCIA should work the better, the more asymmetric the collision system is.

\section{Conclusion}

We have formulated the semiclassical impulse approximation for $K-K$ charge transfer in a way that has made numerical calculations feasible, without further approximations. Numerical results for collisions of protons with $\mathrm{Ne}$ and $\mathrm{Ar}$ are reported and are found to be in good agreement with recent experiments. The comparison with results obtained when an additional peaking approximation is used, shows an agreement within $20 \%$ for systems with $Z_{1} / Z_{2} \lesssim 0.1$ and $v / v_{K} \gtrsim 1$ except for very large $b$ when $v \approx v_{K}$ or $b \approx 0$ for $v / v_{K} \gg 1$. A part from simplifying the numerical work this peaking approximation allows also for the capture to excited projectile states without any complication.

By comparing with experiment we found that the region of validity of the impulse approximation roughly corresponds to that of the first Born approximation for ionisation, as is expected since both theories are first-order theories in the projectile field, while containing the target field to all orders in the interaction. Thus the SCIA is not only valid for high velocities as has been claimed, but it reproduces the 
data also at low velocities provided the collision system is sufficient asymmetric. In the case of a carbon target as well as for $\mathrm{Ne}$ at $v / v_{K} \lesssim 0.5$ we found deviations from experiment which are also present for ionisation and which are probably, at least partly, due to the insufficiency of hydrogenic wavefunctions for these light systems. Unfortunately the present formulation (of Sect. 3) is not suited to treat this problem in more detail, as we exploit explicitly the analytical behaviour of the hydrogenic wavefunctions. However, by means of a multipole expansion of the matrix element calculations with more accurate wavefunctions should be feasible.

We should like to thank L. Kocbach and E. Horsdal Pedersen for interesting discussions and $\mathrm{E}$. Horsdal Pedersen for supplying us with unpublished data. We should also like to thank K. Aashamar for the use of his efficient numerical routines.

\section{Appendix A}

We demonstrate the equivalence between the CIS theory and the peaking approximation (4.2) of the SCIA, specifying to the collisions of protons with targets of charge $Z_{2}$. In the CIS theory, the wavefunctions appearing in the transition amplitude

$a_{f i}=-i \int_{-\infty}^{\infty} d t\left\langle\psi_{f}^{(-)}\left|V_{i}\right| \phi_{i}\right\rangle$

are chosen to include the Coulomb phases

$$
\begin{aligned}
& \psi_{f}^{(-)}=\psi_{f}^{P} e^{-i \eta_{2} \ln \left(v R+v^{2} t\right)} \\
& e^{\pi \eta_{2} / 2} \Gamma\left(1+i \eta_{2}\right)_{1} F_{1}\left(-i \eta_{2}, 1,-i(v r+\mathbf{v} \mathbf{r})\right) \\
& \Phi_{i}=\psi_{i}^{T} e^{i \eta_{2} \ln \left(v^{\prime} R-v^{2} t\right)} .
\end{aligned}
$$

There, $\eta_{2}=Z_{2} / v, \psi_{f}^{P}$ and $\psi_{i}^{T}$ are the final and initial electronic eigenstates, and the internuclear potential $Z_{2} / R$ is included in the Hamiltonian. $\psi_{f}^{(-)}$is obtained by inserting the ansatz $\psi_{f}^{P} L_{f}$ into the Schrödinger equation and neglecting a gradient term in the resulting equation for $L_{f}$ which is a small correction for large $v[11] . \Phi_{i}$ is a solution of the Schrödinger equation where the projectile interaction $V_{1}$ is dropped. The potential $V_{i}$ in (A.1) is thus equal to $V_{1}$ while the internuclear potential only creates the logarithmic phases in (A.2). The reason for this choice is that any phase in $\psi_{f}^{(-)}$vanishes for $t \rightarrow \infty$ (i.e., $\psi_{f}^{(-)} \rightarrow \psi_{f}^{P}$ ), thus compensating for the effect of the projectile on the target at infinite separation.

Inserting (A.2) into (A.1) and noting that $\exp \left(i \eta_{2} \ln \left(v R-v^{2} t\right)\right) \cdot \exp \left(i \eta_{2} \ln \left(v R+v^{2} t\right)\right)=(b v)^{2 i \eta_{2}}$ is independent of time in the straight-line case, we ob- tain with $\psi_{f}^{P}=\psi_{f}^{P}(\mathbf{r}-\mathbf{R}) \exp \left(-i E_{f}^{p} t-i v^{2} t / 2+i \mathbf{v} \mathbf{r}\right)$

$a_{f \mathrm{i}}=-i(b v)^{2 i \eta_{2}} e^{\pi \eta_{2} / 2} \Gamma\left(1-i \eta_{2}\right) \int_{-\infty}^{x} d t e^{i\left(E_{f}^{P}-E_{i}^{T}+z^{2} / 2\right) t}$

$\left\langle\psi_{f}^{P}(\mathbf{r}-\mathbf{R}){ }_{1} F_{1}\left(-i \eta_{2}, 1,-i(v r+\mathbf{v} \mathbf{r})\right) e^{i \mathbf{v r}}\left|V_{1}\right| \psi_{i}^{T}\right\rangle$.

This expression agrees with the peaking result (4.2) of Bransden and Cheshire [17] apart from the phase $(b v)^{2 i \eta_{2}}$. This phase-factor is the one that is also obtained from an eikonal-type approach [28], relating scattering angle to impact parameter. If one instead calculates the transfer probability according to (5.2), it drops out and the two approaches are identical.

\section{Appendix B}

We derive the capture probability into arbitrary excited states of the projectile by means of the peaking approximation. As derived in [20], the transfer probability into a state $\psi_{f}^{p}$ is given by

$a_{f i}^{I^{p}}=i Z_{1} / v(2 / \pi)^{\frac{1}{2}} \int \frac{d \mathbf{s}}{s^{2}} e^{-i \mathbf{s b}}$

$\left\langle\psi_{q_{o<}, \mathbf{e}_{2},}^{T}\left|e^{i \mathbf{s r}}\right| \psi_{i}^{T}\right\rangle \int_{\infty}^{\infty} d T e^{-i q T} \psi_{f}^{* P}(-\mathbf{R})$

with $T=v t$ and $q=s_{z}-\Delta E / v-v / 2$. We use hydrogenic wavefunctions

$\Psi_{n l m}(\mathbf{R})=R_{n l}(R) Y_{l m}\left(\cos \vartheta_{R}, \varphi_{R}\right)$

and choose the $z$-axis along $\mathbf{v}$ such that $\cos \vartheta_{R}=T / R$ and $\varphi_{R}=0$ for the straight-line path. By using the series expansion of the confluent hypergeometric function ${ }_{1} F_{1}\left(-n+l+1,2 l+2,2 Z_{1} R / n\right)=\sum_{\mu=0}^{n-l-1} x_{\mu} R^{\mu}$ which appears in the radial function $R_{n i}(R)$, we can write

$$
\begin{aligned}
& I_{n l m}=\int_{-\infty}^{\infty} d T e^{-i q T} \psi_{f}^{* P}(-\mathbf{R}) \\
& =c_{n l m} \sum_{\mu=0}^{n-l-1} x_{\mu}(-1)^{\mu} \frac{d^{\mu}}{d\left(Z_{1} / n\right)^{\mu}} S_{l m}
\end{aligned}
$$

with

$S_{l m}=\int_{-\infty}^{\infty} d T e^{-i_{q} T} R^{l} e^{-Z_{1} R / n} P_{l}^{m}(T / R)$

$c_{n l m}=\frac{2^{l} Z_{1}^{3 / 2+l}}{n^{2}+l(2 l+1) !}\left[\frac{(n+l) !(l-m) !(2 l+1)}{(n-l-1) !(l+m) ! \pi}\right]^{\frac{1}{2}}(-1)^{l}$

and the $Z_{1}$-dependence of $q$ has to be ignored in the differentiation by $Z_{1}$. Considering first the case $m \geq 0$ and making use of the recurrence relations of the 
Legendre polynomials

$$
\begin{aligned}
& P_{l}^{m}(T / R)=-\frac{1}{(l-m)(l+m+1)} \\
& {\left[P_{l}^{m+2}(T / R)+2(m+1) T / b P_{l}^{m+1}(T / R)\right], m \leq l-2}
\end{aligned}
$$

one can derive corresponding relations for the integrals $S_{l m}$ :

$$
\begin{aligned}
& S_{l m}=-\frac{1}{(l-m)(l+m+1)} \\
& {\left[S_{l, m+2}+2 i(m+1) / b \frac{d}{d q} S_{l, m+1}\right], m \leq l-2 .}
\end{aligned}
$$

Thus, it remains only to calculate the two first integrals $S_{l l}$ and $S_{l, l-1}$. In these cases, the Legendre functions are given by

$$
\begin{aligned}
& P_{l}^{l}(T / R)=(-1)^{l}(2 l-1) ! !(b / R)^{l} \\
& P_{l}^{l-1}(T / R)=(-1)^{l-1}(2 l-1) ! ! T b^{l-1} / R^{l} .
\end{aligned}
$$

From this, it follows

$$
\begin{aligned}
& S_{l l}=2(-1)^{l}(2 l-1) ! ! b^{l+1} \frac{Z_{1} / n}{\left(\left(Z_{1} / n\right)^{2}+q^{2}\right)^{\frac{1}{2}}} \\
& K_{1}\left(b \sqrt{\left.\left(Z_{1} / n\right)^{2}+q^{2}\right)}\right. \\
& S_{l, l-1}=-i / b \frac{d}{d q} S_{l l}
\end{aligned}
$$

and the other integrals entering in (B.3) can be evaluated from (B.5). Due to the symmetry properties of the spherical harmonics $Y_{l,-m}=(-1)^{m} Y_{l m}^{*}$ one has simply for negative $m: I_{n l,-m}=(-1)^{m} I_{n l m}$.

Into $I_{n l m}$ enters thus only the modified Bessel function $K_{1}$ and its derivatives. With the help of the relations

$K_{1}^{\prime}(x)=-K_{0}(x)-\frac{1}{x} K_{1}(x)$

$K_{0}^{\prime}(x)=-K_{1}(x)$,

$I_{n i m}$ can be reduced to the functions $K_{0}$ and $\mathrm{K}_{1}$ alone. The evaluation of the transition amplitude (A.1) proceeds then in complete analogy to the $1 s$ case.

\section{References}

1. Toburen, L.H., Nakai, M.Y., Langley, R.A.: Phys. Rev. 171, $114(1968)$

2. Bayfield, J.E.: Phys. Rev. 185, 105 (1969)

3. Rødbro, M., Horsdal Pedersen, E., Cocke, C.L., Macdonald, J.R.: Phys. Rev. A 19, 1936 (1979)

4. Macdonald, J.R., Cocke, C.L., Eidson, W.W.: Phys. Rev. Lett. 32, 648 (1974)

5. Cocke, C.L., Gardner, R.K., Curnutte, B., Bratton, T., Saylor, T.K.: Phys. Rev. A 16, 2248 (1977)

6. Cocke, C.L., Macdonald, J.R., Curnutte, B., Varghese, S.L., Randall, R.: Phys. Rev. Lett. 36, 782 (1976)

7. Horsdal Pedersen, E., Folkmann, F., Pedersen, N.H.: preprint (1979) and private communications

8. McDowell, M.R.C., Coleman, J.P.: Introduction to the Theory of Ion-Atom Collisions. Chaps. 6-8. Amsterdam: North-Holland 1970

9. Dettmann, K., Leibfried, G.: Z. Physik 218, 1 (1969)

10. Kramer, P.J.: Phys. Rev. A6, 2125 (1972)

11. Cheshire, I.M.: Proc, Phys. Soc. 84, 89 (1964)

12. Belkić, Dž., Gayet, R., Salin, A.: Phys. Rep. 56, 279 (1979)

13. Belkić, Dž.: J. Phys. B 10, 3491 (1977)

14. Chan, F.T., Eichler, J.: Phys. Rev, A 20, 1841 (1979)

15. McDowell, M.R.C.: Proc. Roy. Soc. A 264, 277 (1961)

16. Briggs, J.S.: J. Phys. B 10, 3075 (1977)

17. Bransden, B.H., Cheshire, I.M.: Proc. Phys. Soc. 81, 820 (1963)

18. Coleman, J.P., McDowell, M.R.C., van den Bos, J., de Heer, F.J.: Physica 32, 1164 (1966)

19. Bates, D.R.: Proc. Roy. Soc. A 247, 294 (1958)

20. Amundsen, P.A., Jakubaßa, D.H.: J. Phys. B Lett. (in press)

21. Briggs, J.S., Dubé, L.: J. Phys. B 13, 771 (1980)

22. Bang, J., Hansteen, J.M.: K. Dan. Vidensk. Selsk., Mat. Fys. Medd. 31, 1 (1959)

23. Amundsen, P.A.: J. Phys. B 11, 3197 (1978)

24. Dettmann, K.: Springer Tracts in Modern Physics. Vol. 58, p. 119. Berlin, Heidelberg, New York: Springer 1971

25. Winter, H., Bloemen, E., de Heer, F.J.: J. Phys. B 10, L599 (1977)

26. Nikolaev, V.S.: Sov. Phys. JETP 24, 847 (1967)

27. Aashamar, K., Amundsen, P.A.: Abstracts of Contributed Papers $\mathrm{XI}^{\text {th }}$ ICPEAC, Kyoto. p. 688. Japan: Society for Atomic Collision Research (1979), and submitted for publication

28. McCarroll, R., Salin, A.: J. Phys. B 1, 163 (1968)

D.H. Jakubaßa-Amundsen

P.A. Amundsen

Institute of Physics

University of Oslo

Postboks 1049 Blindern

N-Oslo 3

Norway 